\title{
HADAMARD DESIGNS
}

\author{
EDWARD SPENCE ${ }^{1}$
}

\begin{abstract}
It has already been shown, using a combinatorial argument, that a Hadamard design with each letter repeated once and only once can exist for 2, 4 and 8 letters only. In this paper the same result is proved by a different method which utilizes the underlying algebraic structure of such a Hadamard design.
\end{abstract}

A Hadamard design is a square array of letters which commute in pairs, and to which signs are attached, so that the scalar product of any two distinct rows, considered as vectors, is zero. In [1] Hadamard designs on $n$ letters (or $n$-letter designs) were studied. These are Hadamard designs with $n$ distinct letters where each letter occurs once and only once in each row and column. It was shown that such a design could exist for $n=2,4$ or 8 only.

Clearly, if $H$ is an $n$-letter design, we may suppose that the sign associated with each element in the first row and column is positive (by changing the sign of each element in a row or column, if necessary) and that the first row and column are identical (interchange rows and columns, if necessary). Suppose therefore that $H$ satisfies the above conditions on the letters $a_{1}, a_{2}, \cdots, a_{n}$ and write $-H$ for the design obtained by changing the sign of every letter in $H$. Then we have the following

\section{LEMMA 1.}

$$
\left[\begin{array}{rr}
H & -H \\
-H & H
\end{array}\right]
$$

is the multiplication table of a loop $L$ of order $2 n$ with elements $a_{1}, a_{2}, \cdots$, $a_{n},-a_{1},-a_{2}, \cdots,-a_{n}$.

Proof. Since each letter $a_{1}, a_{2}, \cdots, a_{n}$ occurs once and only once in each row and column of $H$, the array (1) is certainly a latin square. Also,

Received by the editors March 9, 1971.

AMS 1970 subject classifications. Primary 05B20; Secondary 20 N05.

Key words and phrases. Hadamard design, Cayley numbers.

${ }^{1}$ This paper was written while the author held a Visiting Lectureship at the University of Illinois, Urbana, Illinois 61801.

(c) American Mathematical Society 1972 
since its first row and column are identical, it has an identity, and hence is a loop.

In the case $n=2$ it is easy to verify that $L$ is a cyclic group of order 4 and so for the rest of the paper we assume $n>2$.

Suppose that $a_{1}$ is the identity of $L$ and write $a_{1}=1$. It is immediate from (1) that $(-1) a_{i}=-a_{i}=a_{i}(-1)$ and $(-1)^{2}=1$, and hence $\{1,-1\} \subseteq$ $Z$, the center of $L$. Also, from the orthogonality of distinct rows of $H$, we have the following condition:

(2) if $a_{i} \neq \pm a_{j}, a_{k} \neq \pm a_{l}$, then $a_{i} a_{k}=a_{j} a_{l} \Rightarrow a_{j} a_{k}=-\left(a_{i} a_{l}\right)$.

Consequences of (2).

I. $Z=\{1,-1\}$. For $1 a_{i}=a_{i} 1$ and if $a_{i} \neq \pm 1$, (2) implies that $a_{i}^{2}=-1$. Thus if $a_{i} x=x a_{i}$ for all $x \in L$, we must have for some $x \neq \pm 1, \pm a_{i}$ (such exists since $2 n \geqq 6), a_{i}^{2}=-x^{2}=1$, i.e. $a_{i}= \pm 1$ which proves the assertion.

II. If $x, y \in L$, then $x y \neq y x \Rightarrow x y=-y x$. Since $x y \neq y x$ we may suppose

$$
x \neq \pm 1, y \neq \pm 1 \text { and } x \neq \pm y \text {. }
$$

Given $x, y \in L$ there exists a unique $t \in L$ such that $x y=t x$, whence, since $x \neq \pm t$, as is easily shown from (3), $t y=-x^{2}=1$. However $(-y) y=1$ and cancellation yields $t=-y$, i.e. $x y=-y x$.

III. Any two elements of $L$ generate a subgroup. To prove this all we need verify are the following associative laws

(a) $x(x y)=x^{2} \cdot y$,

(b) $(x y) x=x(y x)$,

(c) $(y x) x=y \cdot x^{2}$.

Since these are trivial when $x= \pm 1$ or $y= \pm 1$ or $x= \pm y$ we may assume that none of these equalities is satisfied. Write $x(x y)=1 \cdot z$ so that, by (2), $x y=-x z=x(-z)$. Cancellation gives $y=-z$, i.e. $z=-y$ so that $x(x y)=-y=x^{2} y$ which proves (a). (b) and (c) are proved using II and (a) above.

IV. If $x y \neq y x$ then $x$ and $y$ generate a quaternion group. For $x y \neq y x \Rightarrow$ $x y=-y x$ by II, and the result follows using III.

V. If $x(y z)=(x y) z$ then $x, y$ and $z$ generate a subgroup. Suppose that

(4) $u, v, w \notin Z$ and all lie in different cosets of $Z$ in $L$.

There exists a unique $t \in L$ such that $u v=t w=v(-u)$, and hence (2) yields $v w=-t(-u)=-u t$, and $u(v w)=u(-u t)=-u^{2} t$ (by III) $=t$ since $u \notin Z$. But $(u v) w=(t w) w=-t$, i.e. (uv)w=-u(vw) if $u, v, w$ satisfy (4). Thus if $(x y) z=x(y z)$, then at least one of $x, y, z \in Z$ or $x= \pm y$ or $x= \pm z$ or $y= \pm z$. It follows from III that $x, y$ and $z$ generate a subgroup.

Collecting all this information together, we have

THEOREM 2. The loop L defined in Lemma $1(n>2)$ satisfies the following 
conditions:

(i) The center $Z$ has order two and elements $1,-1$ where $(-1)^{2}=1$, $1 \neq-1$.

(ii) If $x \notin Z$, then $x^{2}=-1$.

(iii) If $x y \neq y x$ then $x y=-y x$ and $x, y$ generate a quaternion group.

(iv) If $x(y z)=(x y) z$, then $x, y, z$ generate a subgroup.

However, it is known that a loop satisfying the conditions of Theorem 2 must either be a quaternion group or a Cayley loop [2, Theorem 7.2 and the remarks following]. Since a quaternion group has order 8 and a Cayley loop has order 16 , it is immediate that $n=4$ or 8 .

In the opposite direction, it is straightforward to verify that the designs obtained from these loops as in (1) are $n$-letter designs. Consequently,

THEOREM 3. There are n-letter designs only for $n=2,4$ or 8 .

\section{REFERENCES}

1. Jennifer Wallis, Hadamard designs, Bull. Austral. Math. Soc. 2 (1970), 45-54. MR 41 \#3304.

2. R. H. Bruck, A survey of binary systems, Ergebnisse der Mathematik und ihrer Grenzgebiete, N.F., Band 20, Springer-Verlag, Berlin, 1958. MR 20 \#76.

Department of Mathematics, University of Glasgow, Glasgow, W.2., Scotland 